\title{
A Method for Dynamic Configuration of a Cognitive Radio
}

\author{
Troy Weingart, Douglas C Sicker, and Dirk GrunDI6TPIQUTION STATEMEAT A \\ Department of Computer Science \\ $430 \mathrm{UCB}$ \\ Approved for Public Pelease \\ Distribution Unlimited
}

Boulder, CO 80309-0439

Troy.Weingart, Douglas.Sicker, Dirk.Grunwald@colorado.edu

303-492-7514

\begin{abstract}
-
Cognitive radios offer a broad range of opportunities for improving the use and utilization of radio frequency spectrum, they also offer a host of exciting prospects in networking research. This includes the creation of radio networks that can reconfigure their operation based on application requirements, policy updates and environmental conditions. Such reconfiguration requires an understanding of cross-layer interactions within the network protocol stack. It also requires the development of algorithms to determine when such reconfigures should be made, and additionally, the potential impacts of these changes on the radio network. In this article, we describe how cognitive radios can be used to create dynamic wireless networks. Such networks can quickly adapt to the needs of users as well as to changes in the environment. We describe how parameters at the application, physical, data link and network layers interact and how desirable configurations of these parameters can be determined. We then describe a technique that uses these configurations in the creation of an adaptive model for a cognitive radio.

"The views expressed in this article are those of the author and do not reflect the official policy or position of the United States Air Force, Department of Defense, or the U.S. Govermment."
\end{abstract}

\section{INTRODUCTION}

The design and implementation of wireless devices is undergoing a substantial transition. While traditionally radio devices had a fixed design and configuration, emerging designs are allowing for much more mutability in both design and configuration. Part of this change has been ushered in by the advent of software defined radio (SDR), a radio with much of its functionality implemented in software [1]. For example, unlike a traditional radio, an SDR might have a modulation scheme that is instantiated in software. A more recent development has been the advent of cognitive radios. These devices are able to reason about their environment and determine when configuration changes are required. Together with the reconfigurable capabilities of SDR, a cognitive radio might dynamically reallocate spectrum or reconfigure itself in response to changes in application demand, operational policy and/or environmental conditions.

Cognitive radios offer a broad range of opportunities for improving the use and utilization of radio frequency spectrum, they also offer a host of opportunities in networking research. This includes the creation of radio networks that can reconfigure their operation based on application requirements (e.g., latency or throughput), environmental conditions (e.g., noise floor) and/or operational policies (e.g., commands to vacate a particular frequency band). Such reconfiguration requires an understanding of cross-layer interactions within the network protocol stack. It also requires the development of algorithms to determine when such reconfigures should be made and the possible impacts of these changes on the radio network. In this article, we describe how cognitive radios can be used to create dynamic wireless networks. Such networks can quickly adapt to users needs as well as to environmental changes.

In developing an adaptive cognitive radio network, it is necessary to understand the implications of varying parameters at the physical, data link and network layers. For example, while it might seem intuitive to increase the transmit power of a radio to ensure that it is heard by the intended recipient, this increase could also harm the communication of other nodes in the area. Furthermore, it might not be beneficial to instantiate a forward error correction scheme on a channel with low error rates. Simply put, it is necessary to understand the implications of varying the parameters within a radio. While gains might well be had by increasing power, unintended issues might suggestion alternative configurations. These issues include decisions about when and how to change configurations, how these changes are propagated throughout the $\mathrm{CR}$ network, and how much time can be spent computing a change in configuration. While these are all important research questions, this article focuses on the development of a predictive model for CRs.

A number of techniques might be applied when determining the potential interaction of input variables and output responses. In this article, we apply Design of Experiments (DOE), this technique requires the identification of factors (inputs to an experiment with differing values or levels) and responses (outputs of the experiment, observations or measures). A series of experiments is run with permutations of the levels of the factors and the responses are recorded. DOE, through statistical methods, is able to identify the significant factors or combinations of factors that impact the response of interest and produce a model for prediction of the response.

This article is organized as follows. Section II describes the background and related work in cognitive networks and 
TABLE I

FACTORS AND RESPONSES FOR 802.11 WIRELESS TEST

\begin{tabular}{|l|l|c|c|}
\hline Factor & Units & Levels & Response \\
\hline \hline Frame Size & Bits & 2048,18432 & Latency \\
Bit Rate & Mbps & 1,2 & Latency \\
Transmit Power & mWatts & 5,100 & Latency \\
\hline
\end{tabular}

network and provides a case study to illustrate how such a network might operate [14]. It is also worth noting that the standards communities are focusing on cognitive radios. The IEEE 802.22 group is developing a wireless standard for the use of cognitive radios to utilize spectrum in geographically separated and vacant TV bands [15]. Also in the IEEE, the P.1900 workgroup is examining the general issue of spectrum management in next generation radio networks.

\section{DESIGN of EXPERIMENTS}

The power of cognitive radio is drawn from its ability to reconfigure in response to a change in the radio frequency environment or a change in the requirements of an application running on the cognitive network. Central to developing any technique for intelligently reconfiguring the cognitive network is a solid understanding of how an individual radio's settings can affect its performance. We sought an approach that would help us determine which settings affect a CR's performance, and settled on a statistical process called Design of Experiments (DOE). DOE is set of tools and methods for determining cause and effect relationships within a system or process [16]. Traditionally, DOE has been used in the process industry to optimize product yield or to maximize production line efficiency. In our case, we use DOE to help determine which configuration of the CR's parameters will have the most positive impact on performance.

DOE is a process that involves a number of steps. The first step is to identify those factors (i.e., inputs to an experiment) that you believe will have an affect on the response (i.e., output of the experiment). Factors will have different levels or values, for example, an 802.11 wireless card may have the capability to transmit at two different power settings. Specifically, in this simulation the factor, transmit power, has the levels of 32 and $100 \mathrm{mWatts}$. Whereas the response is a single value that represents a metric, observation, or measure. In our wireless card example, we measure latency, the response, across a noisy link at each of the levels of our factor. Table I shows a set of factors for an experiment wherein we wish to determine which factors, or interactions between factors, have the most significant impact on latency. An interaction occurs when a factor at one level does not produce the same response at each level of another factor (i.e., latency is not consistent when power is fixed at $32 \mathrm{~mW}$ and Bit Rate varies from 1 to $11 \mathrm{Mbps}$ ). Once the factors, their levels, and the responses are determined you are ready to move on to the next step.

Next we run a set of experiments that iterate through all the combination of factors at each of their levels. Our wireless example requires eight experiments to encompass 
TABLE II

RESULTS FROM 802.11 WIRELESS TEST

\begin{tabular}{|c|r|r|r|r|}
\hline $\begin{array}{c}\text { Run } \\
\text { Order }\end{array}$ & $\begin{array}{r}\text { Frame } \\
\text { Size }\end{array}$ & $\begin{array}{r}\text { Bit } \\
\text { Rate }\end{array}$ & $\begin{array}{r}\text { Transmit } \\
\text { Power }\end{array}$ & Latency \\
\hline \hline 1 & 2048 & 1 & 5 & 0.01430 \\
2 & 18432 & 1 & 5 & 0.01257 \\
3 & 2048 & 2 & 5 & 0.00660 \\
4 & 18432 & 2 & 5 & 0.00570 \\
5 & 2048 & 1 & 100 & 0.01453 \\
6 & 18432 & 1 & 100 & 0.01276 \\
7 & 2048 & 2 & 100 & 0.00660 \\
8 & 18432 & 2 & 100 & 0.00580 \\
\hline
\end{tabular}

TABLE III

ANOVA FOR LATENCY

\begin{tabular}{|l||l|l|c|r||r|}
\hline Source & $\begin{array}{l}\text { Sum of } \\
\text { Squares }\end{array}$ & df & $\begin{array}{r}\text { Mean } \\
\text { Square }\end{array}$ & $\begin{array}{r}\text { F } \\
\text { Value }\end{array}$ & $\begin{array}{l}\text { P Value } \\
\text { Prob }<\text { F }\end{array}$ \\
\hline \hline Madel & $1.119 \mathrm{E}-4$ & 6 & $1.865 \mathrm{E}-5$ & 6472 & $<0.05$ \\
A-FrameSize & $3.233 \mathrm{E}-6$ & 1 & $3.233 \mathrm{E}-6$ & 1122 & $<0.05$ \\
B-BitRate & $1.082 \mathrm{E}-4$ & 1 & $1.082 \mathrm{E}-4$ & 37537 & $<0.05$ \\
C-TransmitPower & $2.753 \mathrm{E}-8$ & 1 & $2.75 \mathrm{E}-8$ & 10 & 0.1992 \\
AB & $4.499 \mathrm{E}-07$ & 1 & $4.499 \mathrm{E}-7$ & 156 & 0.051 \\
AC & $9.643 \mathrm{E}-10$ & 1 & $9.643 \mathrm{E}-10$ & 0.335 & 0.666 \\
BC & $1.607 \mathrm{E}-8$ & 1 & $1.607 \mathrm{E}-8$ & 5.573 & 0.255 \\
\hline Residual & $2.883 \mathrm{E}-9$ & 1 & $2.883 \mathrm{E}-9$ & & \\
Cor Total & $1.119 \mathrm{E}-4$ & 7 & & & \\
\hline \multicolumn{7}{|c|}{$R^{2}: 0.99$} \\
\hline
\end{tabular}

all of the possible configurations. Table II shows the set of experiments and the observed latency for each trial. With a simple example such as ours, you may be able to determine the best configuration through simple inspection of the results. However, when there are many factors, levels, and responses optimization by inspection becomes a time consuming, error prone and difficult task.

The next step in our analysis is to make use of statistical methods to help us identify the significant factors or interactions that impact our response, in this case latency. DOE makes use of Analysis Of Variance (ANOVA) to indicate which factors or interactions most impact the response. Rather than provide a primer on the statistics at work in the ANOVA, an interpretation of the results better serves our purposes. Table III is the ANOVA generated for the experimental runs given in Table II. The sum of squares for the model and residual are shown in the first column of the ANOVA table. The next column is the degrees of freedom associated with the sum of squares. Next is the mean square, or the sum of squares divided by the degrees of freedom. The ratio of the mean squares of the model over the mean squares of the residual forms the next column, and is referred to as the $F$ value. The $F$ value is compared to the reference distribution for $F$, in order to determine the probability of observing this result due to error. In this example, we are using the reference distribution for a 95 percent confidence ratio. If you look closely at the table you'll see that Frame Size and Bit Rate and their interaction are the factors that, according to their high $F$ value and their probability for error, are the most significant factors impacting latency (those factors and interactions with a $P$ Value Prob $<$ $F$ of 0.1 or less are statistically significant).

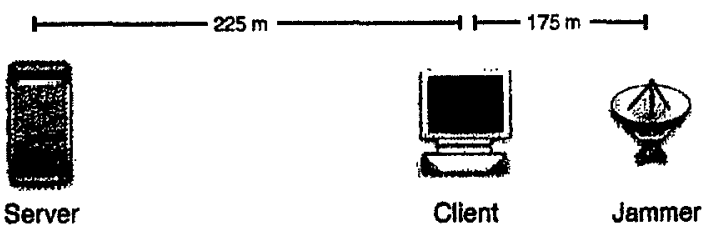

Fig. 1. Simulation Configuration

Finally, we should look at the model behind the ANOVA. The model is a mathematical equation used to predict the response given a set of inputs. In the general case, the equation is of the form given in (1). Where $Y$ is the response and $\beta_{0}$ is the intercept and $\beta_{1}$ is the coefficient for the input factor, $X_{1}$. The larger the coefficient the greater the effect on the prediction. The equation for latency in our simple example is given below after reducing the model to only the significant factors (FrameSize, DataRate, and their interaction). See equation (2). We can plug values into this equation and arrive at a predicted latency. To get an estimate of latency for a given configuration of the $C R$ we need only plug values into this equation. For a frame size of 2048 and a bit rate of $2 \mathrm{Mbps}$ (entered as 2000000 bits) we get a value of 0.006585 , which is very close to the observed response given in Table II (note, power is not included because our ANOVA did not indicate it as a significant factor). It follows that $R^{2}$, a measure of how well a regression line approximates the real data points, is 0.99 (see Table III). Statistically speaking, the model for our simple example provides an almost perfect predictor.

$$
\hat{Y}=\beta_{0}+\beta_{1} X_{1}+\beta_{2} X_{2}+\ldots+\beta_{12 \ldots n} X_{1} X_{2} \ldots X_{n}
$$

$L a t=0.0226-1.644 \mathrm{E}-7 A-7.948 \mathrm{E}-9 B+5.789 \mathrm{E}-14 A B$

\section{Determination of Significant Factors}

In this section, we build upon our wireless 802.11 example by introducing a technique for applying DOE to CRs. The intention is not to necessarily provide the optimal solution; rather we offer an approach for realizing improved performance by matching the needs of the wireless system to changes in the radio environment. We first discuss our simulation environment and then continue with the determination of the significant factors.

\section{A. Experimental Setup and Simulation Environment}

In considering the potential complexity of a cognitive network composed of many nodes, we decided to begin by evaluating a simple network. Our scenario consists of a server communicating with a client in the presence of another node (e.g., a noise source or uncooperative node). Figure 1 shows the layout of the client, server, and noise source. We use OPNET Modeler to simulate the effects of changing factor levels on each of the responses. Although OPNET provides an 802.11 wireless module and media access control (MAC) layer, we had to develop our own module to obtain the 
TABLE IV

FACTORS AND LEVELS OF EACH

\begin{tabular}{|l||l|c|}
\hline Factor & Levels & Layer \\
\hline \hline Offered Load & $0.5,1.5,5.5 \mathrm{Mbps}$ & Application \\
Selective Queueing & off/on & Network \\
Automatic Repeat Request (ARQ) & off/on & MAC \\
Framesize & $2048,9216,18432$ bits & MAC \\
Forward Error Correction & off/on & MAC \\
Jammer Noise Level & $1,4,11 \mathrm{~mW}$ & Physical \\
Bit Rate & $1,2,5,5,11 \mathrm{Mbs}$ & Physical \\
Transmit Power & $5,32,100 \mathrm{~mW}$ & Physical \\
\hline
\end{tabular}

TABLE V

RESPONSES

\begin{tabular}{|l||l|}
\hline Response & Units \\
\hline \hline Bit Loss & Percent \\
Latency & Seconds \\
Jitter & Seconds \\
Throughput & bps \\
\hline
\end{tabular}

flexibility we needed. Our newly developed system allows adaptation of the factor levels on a per packet basis.

\section{B. Factors and Responses}

As discussed previously, the first step in DOE analysis is selection of the factors that will have a significant impact on the measure or response. Table IV lists the factors that we choose for our experiment and the levels used for each. Some of these factors are a part of the configuration parameters of the device (e.g., FEC and data rate) and others are conditional parameters to which the device is subjected (e.g., noise level or offered load). We selected a set of factors that included multiple layers of the traditional protocol stack, as indicated by the column labeled Layer. One might think of the set of factors as a set of dials that one might adjust and the various settings for each factor as the positions on that dial. It is this set of dials that an intelligent process running on a CR would use to configure itself in response to a change in application requirements, policy updates or environmental conditions. DOE requires running 2,592 experiments to cover the permutations of the factors through all of their levels.

There are a host of responses that you could use to evaluate the performance of a cognitive network. We choose those listed in Table $\mathrm{V}$ as a representative set of metrics from which to evaluate the performance of a $\mathrm{CR}$.

\section{ANOVA for Throughput and Latency}

Table VI shows the ANOVA resulting from DOE analysis with respect to throughput. This table is limited to the five factors that contribute most significantly to the response. As you can see load is the primary factor in determining throughput followed by the interaction of load and data rate. An $R^{2}$ of 0.976 also indicates that this model closely predicts the response.

Table VII shows the ANOVA resulting from DOE analysis with respect to latency. This table is limited to the six factors that contribute most significantly to the response. As the
TABLE VI

ANOVA FOR THROUGHPUT

\begin{tabular}{|l||l|l|c|r|c|}
\hline Source & $\begin{array}{l}\text { Sum of } \\
\text { Squares }\end{array}$ & dr & $\begin{array}{c}\text { Mean } \\
\text { Square }\end{array}$ & $\begin{array}{r}\text { F } \\
\text { Value }\end{array}$ & $\begin{array}{l}\text { P Value } \\
\text { Prob }<F\end{array}$ \\
\hline \hline Model & $1.77674 \mathrm{E}+16$ & 9 & $1.97415 \mathrm{E}+15$ & 35505 & $<0.0001$ \\
A-Load & $8.70988 \mathrm{E}+15$ & 1 & $8.70988 \mathrm{E}+15$ & 156647 & $<0.0001$ \\
F-DataRate & $3.58178 \mathrm{E}+15$ & 3 & $1.19393 \mathrm{E}+15$ & 21472 & $<0.0001$ \\
H-FEC & $2.57725 \mathrm{E}+13$ & 1 & $2.57725 \mathrm{E}+13$ & 463 & $<0.0001$ \\
AF & $5.28661 \mathrm{E}+15$ & 3 & $1.76220 \mathrm{E}+15$ & 31693 & $<0.0001$ \\
$A^{2}$ & $1.63309 \mathrm{E}+14$ & 1 & $1.63309 \mathrm{E}+14$ & 2937 & $<0.0001$ \\
\hline \multicolumn{5}{|c|}{$R^{2}: 0.976$} \\
\hline
\end{tabular}

TABLE VII

ANOVA FOR LATENCY

\begin{tabular}{|l||r|r|r|r||r|}
\hline Source & $\begin{array}{r}\text { Sum of } \\
\text { Squares }\end{array}$ & df & $\begin{array}{r}\text { Mean } \\
\text { Square }\end{array}$ & $\begin{array}{r}\text { F } \\
\text { Value }\end{array}$ & $\begin{array}{r}\text { P Value } \\
\text { Prob }<\text { F }\end{array}$ \\
\hline \hline Model & 159653581 & 10 & 15965358 & 40559 & $<0.0001$ \\
A-Load & 52483551 & 1 & 52483551 & 133331 & $<0.0001$ \\
D-ARQ & 108251 & 1 & 108251 & 275 & $<0.0001$ \\
F-DataRate & 44813234 & 3 & 14937744 & 37948 & $<0.0001$ \\
AD & 167242 & 1 & 167242 & 424 & $<0.0001$ \\
AF & 61488802 & 3 & 20496267 & 52069 & $<0.0001$ \\
$A^{2}$ & 592501 & 1 & 592500 & 1505 & $<0.0001$ \\
\hline \multicolumn{5}{|c|}{$R^{2}: 0.981$} \\
\hline
\end{tabular}

results indicate, load again is the primary factor in determining throughput followed by the interaction of load and data rate. An $R^{2}$ of 0.981 also indicates that this model closely predicts the response. The following section describes the use of the equations derived from the ANOVA and presents a method for using this equation to configure a $\mathrm{CR}$.

It is worth noting that there are a number of worthwhile questions that are not addressed here. For example, we do not consider how much time a CR can afford to think about its next configuration or how a large CR network gets reconfiguration information to all nodes robustly and efficiently.

\section{DETERMINATION OF RADIO CONFIGURATION}

In this section, we demonstrate how to apply ANOVA-based models for determining a CR configuration in response to changes in policy, environment, or application requirements.

\section{A. The Technique}

One might consider two primary approaches when applying this DOE technique to cognitive radio networks. One is to do an a priori DOE analysis to determine an initial configuration of the radio. This technique is used after the radio is brought up in its environment and an initial set of training experiments is run. This data is used in the DOE analysis and the development of the predictive model. The second option is for the predictive model to be used by the system in real-time; thereby allowing the radio to dynamically reconfigure in reaction to changes in the environment, policy, or application needs. However, when the set of factors and levels are large the time required to generate the model prohibits the $C R$ from running the $D O E$ analysis in a reactive manner. With current CPU power it is likely that DOE analysis will remain an offline computation for the next few years. 
TABLE VIII

VALUES FOR GENERAL EQUATION (3) FOR THROUGHPUT

\begin{tabular}{|l|l||r|c|c|}
\hline Data Rate & FEC & Intercept & \multicolumn{1}{|c|}{$\beta_{0}$} & \multicolumn{1}{|c|}{$\beta_{1}$} \\
\hline \hline $1 \mathrm{Mbps}$ & off & 75086.189 & 0.570302808 & $-8.13359 \mathrm{E}-08$ \\
$1 \mathrm{Mbps}$ & on & 190227.139 & 0.570302808 & $-8.13359 \mathrm{E}-08$ \\
$2 \mathrm{Mbps}$ & off & 259028.107 & 0.686668263 & $-8.13359 \mathrm{E}-08$ \\
$2 \mathrm{Mbps}$ & on & 374169.057 & 0.686668263 & $-8.13359 \mathrm{E}-08$ \\
$5.5 \mathrm{Mbps}$ & off & -313275.539 & 1.272905782 & $-8.13359 \mathrm{E}-08$ \\
$5.5 \mathrm{Mbps}$ & on & -198134.589 & 1.272905782 & $-8.13359 \mathrm{E}-08$ \\
$11 \mathrm{Mbps}$ & off & -490269.329 & 1.474816703 & $-8.13359 \mathrm{E}-08$ \\
$11 \mathrm{Mbps}$ & on & -375128.379 & 1.474816703 & $-8.13359 \mathrm{E}-08$ \\
\hline
\end{tabular}

TABLE IX

VALUES For GENRRAL. EQUATION (4) FOR LATENCY

\begin{tabular}{|l|l||r|r|c|}
\hline Data Rale & ARQ & Intercept & $\beta_{0}$ & $\beta_{1}$ \\
\hline \hline $1 \mathrm{Mbps}$ & off & -41.13091778 & $6.90218 \mathrm{E}-05$ & $4.89916 \mathrm{E}-12$ \\
$1 \mathrm{Mbps}$ & on & -44.40269207 & $7.33154 \mathrm{E}-05$ & $4.89916 \mathrm{E}-12$ \\
$2 \mathrm{Mbps}$ & off & -18.39882403 & $1.34527 \mathrm{E}-05$ & $4.89916 \mathrm{E}-12$ \\
$2 \mathrm{Mbps}$ & on & -21.67059832 & $1.77463 \mathrm{E}-05$ & $4.89916 \mathrm{E}-12$ \\
$5.5 \mathrm{Mbps}$ & off & 21.59021302 & $-2.91794 \mathrm{E}-05$ & $4.89916 \mathrm{E}-12$ \\
$5.5 \mathrm{Mbps}$ & on & 18.31843873 & $-2.48858 \mathrm{E}-05$ & $4.89916 \mathrm{E}-12$ \\
$11 \mathrm{Mbps}$ & off & 25.14024313 & $-3.29398 \mathrm{E}-05$ & $4.89916 \mathrm{E}-12$ \\
$11 \mathrm{Mbps}$ & on & 21.86846884 & $-2.86462 \mathrm{E}-05$ & $4.89916 \mathrm{E}-12$ \\
\hline
\end{tabular}

We present a technique that makes use of the models resulting from our $\mathrm{DOE}$ analysis. The process described relies upon the identification of a set of application goals or requirements. Imagine that we have a streaming video application that requires a sustained throughput of around $3 \mathrm{Mbps}$. In order to reduce our impact on neighboring nodes, we wish to minimize the time that we occupy the channel and our transmit power. In other words, we want our latency and transmit power to be as small as possible while maintaining adequate throughput.

In general, a CR could operate according to the following: (1) The device would perform a set of evaluative probes to characterize its operating environment. (2) It would then take the resulting data and run DOE analysis to derive the relevant models for the responses of interest. In this example, we are interested in throughput and latency. (3) Upon completion of the models, it is possible to generate all potential configurations that meet application and policy goals. (This would be done on a priority basis by eliminating configurations according to primary, secondary, and subsequent goals). In this example, we narrow by throughput first, then by latency, and finally by transmit power. What follows is a simple example illustrating this technique.

$$
\begin{aligned}
& T P=\text { Intercept }+\beta_{0} * L o a d+\beta_{1} * \operatorname{Load}^{2} \\
& L a t=\text { Intercept }+\beta_{0} * L o a d+\beta_{1} * \operatorname{Load}^{2}
\end{aligned}
$$

\section{B. Example}

In the example, the DOE analysis described in Section III is used. The CR has been set up and has run the initial set of experiments to determine the significant factors for the responses of interest. The ANOVA is completed identifying
TABLE $X$

Configurations Meeting 3 Maps Throughiput Goal

\begin{tabular}{|l||c|c|c|c|c|}
\hline $\begin{array}{l}\text { Bit } \\
\text { Rate }\end{array}$ & FEC & ARQ & $\begin{array}{c}\text { Selectivc } \\
\text { Queneing }\end{array}$ & $\begin{array}{c}\text { Frame } \\
\text { Size }\end{array}$ & $\begin{array}{c}\text { Transmit } \\
\text { Power }\end{array}$ \\
\hline \hline 11 Mbs & any & any & any & any & any \\
\hline
\end{tabular}

TABLE XI

Configurations Meeting 3 MBps Throuphput Goal Wille: MiNimizing LaTENCY

\begin{tabular}{|l||c|c|c|c|c|}
\hline $\begin{array}{l}\text { Bit } \\
\text { Rate }\end{array}$ & FEC & ARQ & $\begin{array}{c}\text { Selective } \\
\text { Queueing }\end{array}$ & $\begin{array}{c}\text { Frame } \\
\text { Size }\end{array}$ & $\begin{array}{c}\text { Transmit } \\
\text { Power }\end{array}$ \\
\hline \hline 11 Mbs & any & off & any & any & any \\
\hline
\end{tabular}

the significant factors and the underlying model is generated (see prior section). The model for throughput is given in (3). This equation represents a method for predicting throughput given a radio configuration or when applied in reverse the equation can be used to generate all configurations that are capable of providing a given throughput. This is the first step in determining the set of potential configurations that will meet the primary goal of $3 \mathrm{Mbps}$ throughput. In this scenario, there is a streaming video application running on a cognitive network. This application requires a throughput of $3 \mathrm{Mbps}$. To use the general equation for throughput one needs to apply the proper intercept and coefficients (see Eqn 3). These values are given in Table VIII. For example, a configuration with Data Rate of $5.5 \mathrm{Mbps}$ and FEC set on you would use the values from row 6 . When reducing the number of configurations for the desired throughput, the model only identifies those settings using the $11 \mathrm{Mbps}$ data rate. As one might deduce, the $\mathbf{5 . 5}$ Mbps data rate is not sufficient to meet the stated goal due to protocol overhead and noise in the environment. When substituting $3,000,000$ for load into the equation provided by (3) and Table VIII at the $5.5 \mathrm{Mbps}$ data rate, we get $2,773,419$ (FEC off) and 2,888,560 (FEC on) - rates that are not able to meet the required load. The set of potential configurations is now limited to those with the $11 \mathrm{Mbps}$ data rate (in this case there are 72 configurations remaining, see Table $X$ ).

The next step involves further reducing this set of configurations in order to minimize latency. When applying the equations in (4) and Table IX the set of configurations is reduced by half, to 36 , through elimination of those configurations with Automatic Repeat Request (ARQ) set on. Further reduction of the set of configurations to minimize transmit power, results in those configurations listed in Table XII. The size of this data set could be pruned further if we had not simplified the models for throughput and latency by the top five or six factors. If all the significant factor interactions were included in the model, our technique would identify one best configuration - a data rate of $11 \mathrm{Mbps}$, with FEC set on, ARQ set off, a frame size of 18432 bits, and a transmit power of $5 \mathrm{~mW}$.

\section{ConClusion}

In this article, we have described how cognitive radios can be used to create dynamic wireless networks. Such networks 
TABLE XII

Configurations Meeting 3 Mbps Throuphput Goal While MINIMIZING LATENCY AND TRANSMIT POWER (DATA RATE = $11 \mathrm{MBS,}$ TRANSMIT POWER $=5 \mathrm{MW}$ AND ARQ $=$ OFF)

\begin{tabular}{|c||l|l|l|l|l|}
\hline$\#$ & FEC & $\begin{array}{c}\text { Selective } \\
\text { Queueing }\end{array}$ & $\begin{array}{c}\text { Frame } \\
\text { Size }\end{array}$ & $\begin{array}{c}\text { Predicted } \\
\text { Throughput }\end{array}$ & $\begin{array}{c}\text { Predicted } \\
\text { Latency }\end{array}$ \\
\hline \hline 1 & off & off & 2048 & 3202158 & -29.5869 \\
2 & on & off & 2048 & 3317299 & -29.5869 \\
3 & off & on & 2048 & 3202158 & -29.5869 \\
4 & on & on & 2048 & 3317299 & -29.5869 \\
5 & off & off & 9216 & 3202158 & -29.5869 \\
6 & on & off & 9216 & 3317299 & -29.5869 \\
7 & off & on & 9216 & 3202158 & -29.5869 \\
8 & on & on & 9216 & 3317299 & -29.5869 \\
9 & off & off & 18432 & 3202158 & -29.5869 \\
10 & on & off & 18432 & 3317299 & -29.5869 \\
11 & off & on & 18432 & 3202158 & -29.5869 \\
12 & on & on & 18432 & 3317299 & -29.5869 \\
\hline
\end{tabular}

can be used to quickly adapt to the needs of users as well as to changes in the environment. We described methods for determining how parameters at the physical, data link, network and application layers interact and showed how desirable configurations of these parameters can be determined. We also described a technique that can make use of these configurations in the creation of an adaptive model for a cognitive radio.

\section{ACKNOWLEDGMENT}

The authors would like to thank Gary Yee and John Giacomoni for their assistance in this research.

\section{REFERENCES}

[1] ITU, "Wp8a draft report on sdr," September 2004.

[2] A. Goldsmith and S. Wicker, "Design challenges for energy-constrained ad hoc wireless networks," IEEE Wireless Communications, vol. 9, pp. 8-27, 2002.

[3] C. Barrett, A. Marathe, M. V. Marathe, and M. Drozda, "Characterizing the interaction between routing and mac protocols in ad-hoc nctworks," in MobiHoc '02: Proceedings of the 3rd ACM international symposium on Mobile ad hoc networking \& computing. New York, NY, USA: ACM Press, 2002, pp. 92-103.

[4] R. Jiang, V. Gupta, and C. Ravishankar, "Interactions between tep and the icce 802.11 mac protocol," in Proc. of DISCEX, 2003.

[5] V. Kawadia and P. R. Kumar, "A cautionary perspective on cross layer design," IEEE Wireless Communication Magazine, vol. 12, pp. 3-11, 2005.

[6] K. K. Vadde and V. R. Syrotiuk, "Factor interaction on service delivery in mobile ad hoc networks," IEEE Jourmal on Selected Areas in Communications, vol. 22, pp. 1335-1346, September 2004

[7] K. Vadde and V. Syrotiuk, "Quantifying Factors Affecting Quality of Service in Mobile Ad Hoc Networks," SLMULATION, vol. 81, no. 8, pp. 547-560, 2005. [Online]. Available: http://sim.sagepub.com/cgi/content/abstract/81/8/547

[8] K. K. Vadde, V. R. Syrotiuk, and D. C. Montgomery, "Optimizing protocol interaction using response surface methodology", IEEE Transactions on Mobile Computing, vol. 5, no. 6, pp. 627-639, 2006.

[9] S. Haykin, "Cognitive radio: brain-empowered wireless communications," IEEE Joumal on Selected Areas in Communications, vol. 23, pp. 201-220, 2005.

[10] L. Berlemann, S. Mangold, and B.H.Walke, "Policy-based reasoning for spectrum sharing in cognitive radio networks," in Proc. IEEE DySPAN 2005 , November November 2005 , pp. 1-10.

[11] M. Buddhikot, P. Kolody, S. Miller, K. Ryan, and J. Evans, "Dimsumnet: new directions in wireless networking using coordinated dynamic spectrum access," Proc. IEEE WoWMoM 2005, 2005.
[12] A. Sahai, N. Hoven, and R. Tandra, "Some fundamental limits in cognitive radio," in Allerton Conf. on Commun., Control and Computing, October 2004.

[13] S. Nishra, D. Cabric, C. Chang, D. Willkomm, B. Schewick, A. Wolisz, and $R$. Brodersen, "A real time cognitive radio testbed for physical and link layer experiments," in Proc. IEEE DySPAN 2005, November November 2005, pp. 562-567.

[14] . R. Thomas, L. DaSilva, and A. MacKenzie, "Cognitive networks," in Proc. IEEE DySPAN 2005, November November 2005, pp. 352-360.

[15] C. Cordeiro, K. Challapali, D. Birru, and S. Shankar, "Icee 802.22: the first worldwide wireless standard based on cognitive radios," in Proc. IEEE DySPAN 2005, November November 2005, pp. 328-337.

[16] P. W. M. Anderson, "Doe simplified," 2000. 
